1 Universidade Federal de Minas Gerais (UFMG) Núcleo de Educação em Saúde Coletiva (Nescon) - Belo Horizonte (MG), Brasil.

cardoso@nescon.medicina. ufmg.br

\section{O Brasil na UTI: atenção hospitalar em tempos de pandemia}

\author{
Brazil in the ICU: hospital care in times of pandemic
}

Francisco Carlos Cardoso de Campos' ${ }^{\mathbf{1}}$ Claudia Marques Canabrava'

DOI: 10.1590/0103-11042020E409

\section{Introdução}

Ao avançar sobre o Brasil, a pandemia da Covid-19 gerou uma elevada demanda emergencial e crescente ao Sistema Único de Saúde (SUS), descortinando uma das dimensões mais graves da crise sanitária brasileira: a do seu componente hospitalar. A estrutura assistencial hospitalar brasileira historicamente insuficiente, geograficamente mal distribuída, irregularmente integrada aos sistemas locais e regionais, com indicadores de desempenho contestáveis, além de severamente desgastada pelo subfinanciamento crônico, vê-se então diante da impactante demanda por um grande número de leitos hospitalares (gerais e de Unidades de Terapia Intensiva - UTI) para o cuidado às vítimas do coronavírus, sobretudo as mais graves ${ }^{1-6}$.

A cada leito necessário para atendimento à Covid-19, são também exigidos novos fluxos de organização de acesso, novos equipamentos, especialmente ventiladores mecânicos, uma rede elétrica e de gases capaz de suportar essa sobrecarga, insumos em quantidade e qualidade adequadas e, sobretudo, força de trabalho capacitada para atender ao crescente número de casos complexos e muito graves ${ }^{7}$.

Esse contexto desnuda a fragilidade dos processos regulatórios assistenciais, as decisões equivocadas do mercado mundial com concentração da produção de medicamentos, os equipamentos e insumos médico hospitalares em pouquíssimos países, a realidade de infraestruturas prediais arcaicas em sua maioria, além do desolador cenário das insuficiências de oferta, qualificação e remuneração da força de trabalho, em especial, médica e de enfermagem.

Na conjuntura política nacional, destacam-se o inusitado descaso e o despreparo do governo federal na coordenação dos processos de enfrentamento da Covid-19, associada à inconsequente retórica da falsa valorização da economia ante a proteção sanitária da população, com manutenção das medidas de austeridade fiscal e congelamento de gastos públicos pela Emenda Constitucional $\mathrm{n}^{\circ}$ 95, de 16 de dezembro de $2016^{8}$. Em recente documento elaborado pelo Ministério da Economia, o aprofundamento das políticas liberais é defendido enfaticamente, ao contrário do que seria de se esperar para contrapor ao ambiente econômico recessivo e com taxas elevadíssimas de desemprego e informalidade 9 . Aliás, em meio à pandemia, e se justificando a partir dela, é lançada a Medida Provisória $n^{\circ}$ 905, que altera mais de 86 itens da Consolidação das Leis do Trabalho (CLT), que manifesta intenção de recuperar a economia à 
custa da redução de direitos dos trabalhadores $^{10}$, já extremamente fragilizados pela recessão e desemprego.

A desorganização entre níveis de governo com pouco ou nenhum engajamento solidário e sinérgico provoca instabilidades e desconfianças entre estes e destes com a população. Em meio às intempéries sanitárias e políticas, convive-se com uma situação de carência de informações insuspeitas quanto ao número de casos e de óbitos, que faz supor um grande sub-registro, seja pela insuficiência de testagem, seja pela falta do monitoramento sistemático dos doentes controlados ambulatorialmente e de seus contatos.

A adoção de medidas transparentes, embasadas em evidências científicas e coordenadas, é mandatória, sob pena de agravar o catastrófico quadro sanitário que se antevê: uma epidemia que tende a se tornar endêmica em patamares muito elevados de incidência, associada a um aparato sanitário extremamente combalido do ponto de vista do seu custeio adequado, que pode ser literalmente aniquilado, em futuro próximo, pela continuidade e insistência nas políticas de cunho liberal, centradas no ajuste fiscal e na desregulamentação dos mercados.

Há que se reconhecer que a grave crise não se restringe ao aparato assistencial hospitalar público do SUS. Tem-se, de fato, cerca de $60 \%$ dos estabelecimentos hospitalares privados sem fins lucrativos com duas 'portas de entrada'11. Também convivem, ao menos, duas grandes modalidades de remuneração dos serviços de saúde: uma ‘contratualização' pelo SUS, negociada em valores orçamentários globais vinculados a metas quantitativas e qualitativas, e um sistema de pagamento por procedimentos e atos profissionais (fee for service); esta última modalidade, praticada tanto pelo SUS quanto pelas operadoras de planos e seguros de saúde do chamado subsistema 'suplementar'. Questiona-se fortemente a sustentabilidade econômico-financeira do sistema quando se leva em consideração a espiral de preços dos insumos e equipamentos associada à brusca redução na produção de serviços, pela tentativa de criar uma reserva para o atendimento da demanda emergencial de casos com Covid-19 e/ou pela resistência das pessoas a procurarem os serviços de saúde, pelo evidente risco de contaminação e adoecimento, com reprogramação da assistência.

Dessa maneira, a conjuntura atual coloca em cena o espectro da morte iminente, tanto para os brasileiros quanto para o sistema hospitalar do SUS, público e privado sem fins lucrativos. É nesse contexto complexo e desafiador, profundamente marcado por uma tripla crise - sanitária, política e econômica -, que se pretende levantar alguns pontos para a reflexão sobre a atenção hospitalar neste tempo de convivência com a Covid-19, até que surjam recursos terapêuticos e profiláticos que façam cessar essa demanda adicional que o cuidado aos casos graves suscita.

Obviamente, não se pretende abranger toda a complexidade do tema, mas apresentar alguns achados de interesse no panorama hospitalar do SUS, reconhecendo-se, a priori, a limitação dessa perspectiva analítica situacional, praticada simultaneamente aos acontecimentos. Apesar do risco consciente de apresentar algumas informações que podem se modificar rapidamente na conjuntura, haja vista a rapidez que o quadro sanitário e assistencial tem mudado no decorrer da pandemia, julgou-se oportuno abordar sucintamente os seguintes pontos: uma breve caracterização do parque hospitalar do SUS; as estruturas criadas para enfrentamento da Covid-19, especialmente os leitos de UTI; as iniciativas metodológicas para o cálculo de suas necessidades; os primeiros efeitos sentidos no campo da atenção hospitalar, especialmente as internações de alguns grupos de procedimentos; e, finalmente, um conjunto de proposições para a garantia da sustentabilidade do componente hospitalar do sistema de saúde brasileiro. 


\section{Breve caracterização da rede hospitalar SUS}

Para caracterização da rede hospitalar SUS, foram analisados os dados constantes do Cadastro Nacional de Estabelecimentos de Saúde (CNES), criado em 2003 e implantado progressivamente até ser definitivamente regulamentado em 2015 por meio da Portaria GM/MS n ${ }^{0} 1.646$, de 2 de outubro de $2015^{11,12}$. Apesar de ser constantemente criticado pelas discrepâncias entre a situação real e a cadastrada, sua utilização de forma crítica é incontornável. Dessa maneira, optou-se por associar dados do Sistema de Internação Hospitalar (SIH/SUS) do ano de 2019 para melhor alcance da análise.

O CNES apresentou, na sua competência maio de 2020, 8.018 estabelecimentos com ao menos 1 leito cadastrado. Desse total, 5.889 (73,44\%) registram vínculo com o SUS. Apesar disso, 154 estabelecimentos não apresentam nenhum leito SUS cadastrado e foram descartados da análise. O grupo de 5.735 estabelecimentos com leitos SUS cadastrados foram distribuídos segundo tipo de estabelecimento da seguinte maneira: 1) $69,0 \%$ Hospitais Gerais; 2) 9,8\% Unidades Mistas; 3) 9,4\% Hospitais Especializados; 4) 2,7\% Hospitais Dia Isolados; 5) 1,6\% Pronto-Socorro Geral ou Especializado; 6) 0,3\% Centro de Parto Normal; e 7) 7,2\% 'outros' tipos, a exemplo dos Centros de Apoio Psicossocial (Caps) que, apesar de apresentarem leitos cadastrados, não são estabelecimentos destinados à internação hospitalar propriamente dita.

Em referência à capacidade real de atendimento de internação, inclusive de casos de Covid-19, optou-se por aprofundar o exame do conjunto de 5.306 estabelecimentos com vínculo SUS e ao menos 1 leito SUS cadastrado no CNES, dos 5 primeiros tipos descritos acima. A análise da produção de serviços, por meio das Autorizações de Internação Hospitalar (AIH), informadas no SIH/SUS, para o ano de 2019, pode efetivamente minimizar eventuais omissões dos cadastros e excluir os casos de não funcionamento real dos estabelecimentos.

Dos 5.306 estabelecimentos cadastrados, 813 (15,3\%) não apresentaram nenhuma AIH em 2019, podendo ser considerados, a priori, 'inoperantes e ou inexistentes de fato'. Outros 429 (8,1\%) estabelecimentos apresentaram menos de 120 AIH para o ano de 2019 e Taxa de Ocupação Hospitalar (TOH) SUS menor que $30 \%$, considerados nesta análise como 'estabelecimentos com baixa capacidade instalada de leitos e/ou internação esporádica'. Os demais 4.064 (76,6\%) estabelecimentos foram analisados considerando-se tanto a produção aprovada de AIH ( $\geq 121 \mathrm{AIH} / 2019)$ quanto sua $\mathrm{TOH}$, calculada dividindo-se a permanência apresentada SIH/SUS pelo número de leitos-dia SUS.

Desses 4.064 hospitais, 57 (1,4\%) apresentaram $\mathrm{TOH}=0 \%$, o que foi compreensível considerando que, em sua maioria, trata-se de Hospitais-Dia Isolados e/ou hospitais especializados dedicados ao atendimento oftalmológico, com raros tempos de permanência superiores a 24 horas. Outros 1.729 hospitais (42,5\%) apresentaram $\mathrm{TOH}$ entre $1 \%$ e $30 \%$ e, apesar de apresentarem número superior a $121 \mathrm{AIH} / \mathrm{ano}$, de fato, não executaram internações na mesma proporção que seus leitos cadastrados, ou seja, foram também considerados 'estabelecimentos com baixa capacidade instalada de leitos e/ou internação esporádica'.

Restaram, então, 2.278 hospitais com TOH $>30 \%$ considerados como 'hospitais operantes' e que juntos somaram 284.058 leitos totais cadastrados (competência CNES maio de 2020), sendo 242.618 gerais SUS ( $85,4 \%)$, e, 37.524 leitos complementares cadastrados, sendo 29.688 leitos complementares SUS (79,1\%). Do conjunto de hospitais analisados como 'operantes', as capitais brasileiras contaram com $22,9 \%$ do número de estabelecimentos; $37,4 \%$ dos leitos totais; $38,9 \%$ dos leitos totais SUS; $44,2 \%$ dos leitos de complementares tanto existentes quanto SUS coincidentemente (figura 1). 
Figura 1. Número de estabelecimentos existentes e o número de leitos em hospitais operantes

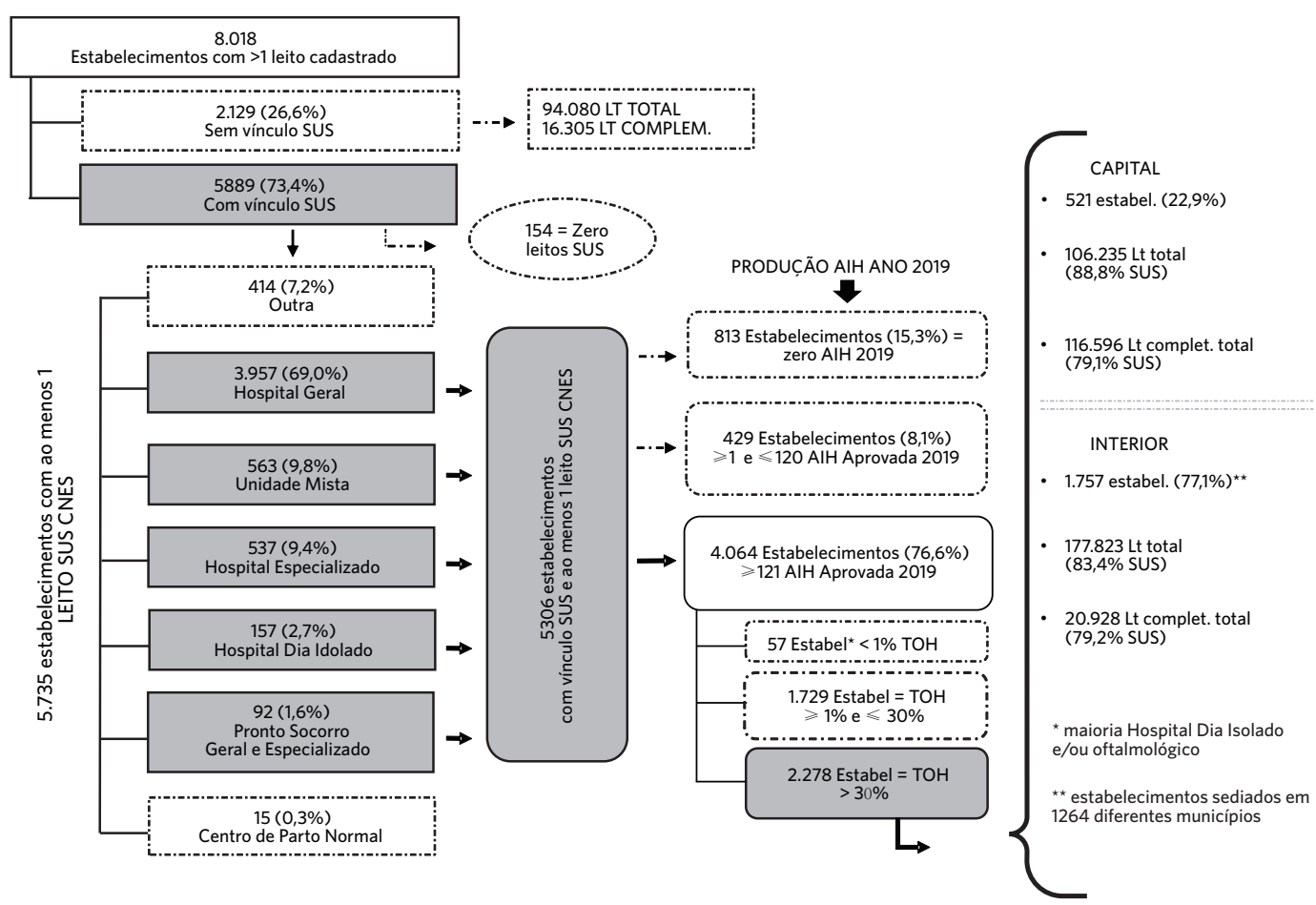

Fonte: Elaboração própria.

Quando analisados os 2.278 estabelecimentos e sua distribuição regional, observou-se que 118 Macrorregiões de Saúde (100\%) e 426 Regiões de Saúde CIR - Comissões Intergestores Regionais (95,1\%) apresentavam ao menos 1 dos hospitais dessa lista. Por outro lado, a despeito das iniciativas de regionalização da saúde, isso significa muito pouco se observadas as grandes distâncias percorridas, tempos de deslocamento e oportunidades de atenção para uma população de cerca de 212 milhões de brasileiros, distribuída nos 5.570 municípios do País ${ }^{13}$.

Do total de 8.018 estabelecimentos com leitos cadastrados, 1.867 não têm vínculo com o SUS e juntos somavam 94.080 leitos totais e 16.305 leitos complementares, para os quais não é possível aplicar a mesma metodologia de análise, tendo em vista não estarem acessíveis as bases de dados de internações do sistema suplementar de saúde no Brasil em um padrão mínimo de abrangência e qualidade dos dados. Se, hipoteticamente, todos esses leitos estiverem em estabelecimentos hospitalares operantes, tal qual se considerou para os hospitais com vínculo SUS, dispor-se-ia, em maio de 2020 no Brasil, de 378.138 leitos totais e 53.829 leitos complementares, em uma proporção de 1,78 leito total e 0,25 leito de UTI a cada mil habitantes. Esses números são por si só alarmantes e evidenciam a insuficiência de leitos efetivos no Brasil3. Ademais, podem surpreender ainda mais se for considerada a diferença significativa de oportunidade de acesso entre os brasileiros com uso exclusivo da infraestrutura hospitalar SUS (78\%) e aqueles assegurados pela saúde suplementar $(22 \%)^{14}$, ampliando consideravelmente as iniquidades sanitárias no País. 


\section{A oferta de leitos de UTI no Brasil}

Dados relativos a julho de 2020 e constantes do CNES indicam a existência de um total de 86.392 leitos complementares, sendo 51,6\% públicos ou contratados pelo SUS e os restantes $48,4 \%$ de propriedade privada típica. Desses leitos, 31.940 são leitos de UTI Adulto e 4.938 de UTI Pediátrica, sendo, respectivamente, $15.322(48,0 \%)$ e $2.669(54,1 \%)$ 'leitos SUS'.

Em março de 2020, por meio da Portaria GM/MS n ${ }^{\circ} 237$, foram incluídos na Tabela de Leitos do CNES, Tipo 03 - Complementar, o Leito 51 - UTI II Adulto - Covid-19 e o Leito 52 - UTI II Pediátrica - Covid-19. Ademais, em julho de 2020, estiveram cadastrados 20.203 leitos Covid-19 Adulto e 729 leitos Covid-19 Pediátricos, sendo, respectivamente, 10.228 (50,6\%) e 200 (27,4\%) SUS. Esse incremento representa $35,3 \%$ de crescimento sobre o total de leitos complementares do período imediatamente anterior à pandemia (fevereiro) e 58,7\% de ampliação sobre o somatório dos leitos de UTI Adulto e Pediátrico existentes em fevereiro de 2020 (35.682 leitos à época). O maior crescimento proporcional ocorreu na Região Sudeste (48,3\%), seguido das Regiões Nordeste (24,7\%), Sul (13,0\%), Norte $(6,0 \%)$ e Centro-Oeste (8,0\%).

A habilitação de leitos de UTI dedicados exclusivamente aos casos de Covid-19 pelo Ministério da Saúde foi regulamentada pela Portaria GM/ $\mathrm{MS} \mathrm{n}^{0} 568$, de 26 de março de $2020^{15}$. Entre abrile agosto de 2020, foram habilitados 12.244 leitos de UTI Covid-19 Adultos e 249 leitos de UTI Covid19 Pediátricas, a partir de 156 diferentes portarias ministeriais. Uma das razões da diferença entre o número de leitos habilitados pelo Ministério da Saúde e aqueles cadastrados como leitos SUS na competência CNES julho 2020 é exatamente o tempo entre a publicação de habilitação e atualização dos dados pelos estabelecimentos e processamento pelo Datasus no CNES. Posto isso, podemos considerar que $100 \%$ dos leitos SUS Covid-19, tanto adulto quanto pediátrico, estão habilitados.
Por outro lado, o protagonismo estadual e dos municípios das capitais brasileiras na organização da oferta de atenção hospitalar no SUS se manifesta, de forma inequívoca, na criação emergencial de novos leitos de UTI para o enfrentamento da pandemia. Evidentemente, em qualquer epidemia ou ameaça à saúde e à segurança nacionais, uma forte coordenação federal das políticas públicas há que ocorrer, não só pela sua maior capacidade de financiamento e mobilização de técnicos e especialistas, além dos complexos processos de acesso e compra de insumos e equipamentos no exterior, mas também pela necessária solidariedade e corresponsabilidade entre os entes federados na gestão do sistema, princípio ademais inscrito na Constituição Federal.

Proporcionalmente, o estado de São Paulo foi o que teve o maior número de leitos de UTI Covid-19 habilitados (23,7\%), seguido de Minas Gerais (9,1\%) e do Rio de Janeiro com (6,9\%). Já a maioria dos estados da região Norte ( 5 das 7 unidades federadas) apresentaram cada qual menos de $1 \%$ do total de leitos de UTI Covid-19 habilitados, o que é compreensível dada a falta de estrutura hospitalar história, mas que, por outro lado, requer atenção para novos investimentos.

Os leitos UTI Covid-19 Adulto SUS representam a maioria dos leitos de UTI Adulto SUS no País (67\%), com destaque para os estados do Mato Grosso (90\%), do Acre (91\%), de Santa Catarina (94\%), do Rio Grande do Norte (107\%), do Ceará (121\%), do Espírito Santo (139\%) e do Piauí (175\%). Talvez essa proporção se justifique melhor pelo incentivo financeiro do que propriamente pela necessidade sanitária.

Do total de leitos Covid-19 habilitados, 79,7\% estão inseridos nos hospitais, 2.278 classificados como 'operantes', sendo 9.761 Covid-19 Adulto e 201 Pediátrico. Isso nos dá a real dimensão dos hospitais com os quais a população 'SUS dependente' pode efetivamente contar e para os quais deve haver maiores investimentos e maior dedicação em monitorá-los, seja do ponto de 
vista de sua sustentabilidade financeira, seja do ponto de vista de seu desempenho e papel na rede de atenção.

A habilitação temporária de leitos de UTI pelo Ministério da Saúde passou a remunerar esses serviços com um valor diário de $\mathrm{R} \$$ 1.600,00. Apesar de representar o dobro do valor que vinha sendo pago para os leitos de UTI de hospitais inscritos nas 'Redes Temáticas Prioritárias de Urgência \& Emergência e da Rede Integrada de Atenção à Gravidez, Parto e Puerpério e crianças até 2 anos (Rede Cegonha)', e de ser quatro vezes o valor pago pelo paciente-dia das internações usuais nas UTI, esse montante de recursos financeiros é bastante inferior ao observado na área privada. Um estudo realizado pelo Grupo IAG, com base em dados de 106 hospitais brasileiros em 2019, encontrou um valor de $\mathrm{R} \$ 1.934,00$ para a diária de UTI ${ }^{16}$. Além disso, em recente debate por videoconferência, um dos autores do estudo revelou que os custos operacionais em 7 hospitais privados apresentam valor atualizado de $\mathrm{R} \$ 2.500,00$, resultante da elevada inflação dos preços de insumos e dos recursos humanos em meio à pandemia ${ }^{17}$.

Não obstante os incentivos financeiros de fomento à habilitação leitos UTI Covid-19, comparados aos valores anteriormente aplicados, tal iniciativa não garante sustentabilidade à rede de hospitais, seja pela sua temporalidade de 90 dias a constar da data de publicação da Portaria $\mathrm{n}^{0}$ 568 , de 26 de março de 2020, seja pelos custos apresentados em outras análises ${ }^{15-17}$.

Quadro 1. Leitos de UTI Covid-19 adulto e pediátrico segundo UF, Brasil, julho de 2020

\begin{tabular}{|c|c|c|c|c|c|c|c|c|c|c|c|c|c|c|c|}
\hline \multirow[b]{2}{*}{ UF } & \multicolumn{5}{|c|}{ Total de leitos (Lt) cadastrados CNES } & \multicolumn{5}{|c|}{ Leitos (Lt) SUS } & \multicolumn{2}{|c|}{ Leitos habilitados Covid* } & \multicolumn{3}{|c|}{ Hospitais Operantes } \\
\hline & $\begin{array}{l}\text { Lt. Comple- } \\
\text { mentares } \\
\text { existentes }\end{array}$ & $\begin{array}{l}\text { Lt. UTI } \\
\text { adulto }\end{array}$ & $\begin{array}{l}\text { Lt. UTI } \\
\text { pediátrica }\end{array}$ & $\begin{array}{l}\text { Lt. UTI } \\
\text { Covid } \\
\text { adulto }\end{array}$ & $\begin{array}{l}\text { Lt. UTI } \\
\text { Covid } \\
\text { pediátrico }\end{array}$ & $\begin{array}{l}\text { Lt. Com- } \\
\text { plementa- } \\
\text { res SUS }\end{array}$ & $\begin{array}{l}\text { SUS_UTI } \\
\text { adulto }\end{array}$ & $\begin{array}{l}\text { SUS_UTI } \\
\text { pediátrica }\end{array}$ & $\begin{array}{l}\text { SUS_UTI } \\
\text { Covid-19 } \\
\text { adulto }\end{array}$ & $\begin{array}{l}\text { SUS_UTI } \\
\text { Covid-19 } \\
\text { pediátrico }\end{array}$ & $\begin{array}{l}\text { Lt. habili- } \\
\text { tado Covid } \\
\text { UTI adulto }\end{array}$ & $\begin{array}{l}\text { Lt. habilitado } \\
\text { Covid UTI } \\
\text { pediátrico }\end{array}$ & $\begin{array}{l}\text { № de } \\
\text { hospitais } \\
\text { operantes }\end{array}$ & $\begin{array}{l}\text { \% Lt. Covid } \\
\text { habilitado } \\
\text { adulto }\end{array}$ & $\begin{array}{l}\% \text { Lt. Covid } \\
\text { habilitado } \\
\text { pediátrico }\end{array}$ \\
\hline \multicolumn{16}{|c|}{ Centro-Oeste } \\
\hline GO & 2.598 & 1.052 & 152 & 545 & 20 & 1.264 & 526 & 93 & 227 & 0 & 312 & & 70 & $36,5 \%$ & \\
\hline MT & 1.517 & 531 & 78 & 373 & 30 & 723 & 198 & 18 & 179 & 20 & 274 & 20 & 38 & $41,6 \%$ & $50,0 \%$ \\
\hline MS & 985 & 389 & 40 & 252 & 3 & 585 & 199 & 33 & 144 & 3 & 194 & 3 & 53 & $82,0 \%$ & $100,0 \%$ \\
\hline DF & 2.729 & 1.117 & 282 & 342 & 10 & 623 & 262 & 76 & 165 & 0 & 175 & 10 & 16 & $100,0 \%$ & $0,0 \%$ \\
\hline \multicolumn{16}{|c|}{ Norte } \\
\hline PA & 2.532 & 719 & 150 & 486 & 44 & 1.553 & 382 & 124 & 290 & 14 & 322 & 14 & 116 & $66,1 \%$ & $100,0 \%$ \\
\hline AM & 1.305 & 285 & 127 & 242 & 15 & 864 & 192 & 108 & 122 & 0 & 191 & 8 & 39 & $73,3 \%$ & $0,0 \%$ \\
\hline RO & 751 & 263 & 31 & 158 & 7 & 415 & 159 & 18 & 48 & 0 & 108 & 7 & 21 & $78,7 \%$ & $100,0 \%$ \\
\hline TO & 530 & 163 & 31 & 91 & 14 & 316 & 73 & 21 & 36 & 12 & 83 & 6 & 15 & $75,9 \%$ & $100,0 \%$ \\
\hline$A C$ & 285 & 58 & 12 & 74 & 0 & 170 & 33 & 11 & 30 & 0 & 50 & & 10 & $100,0 \%$ & \\
\hline AP & 256 & 41 & 10 & 93 & 4 & 89 & 12 & 5 & 0 & 0 & 32 & & 7 & $100,0 \%$ & \\
\hline$R R$ & 148 & 31 & 11 & 25 & 15 & 102 & 17 & 10 & 10 & 5 & 20 & 5 & 6 & $100,0 \%$ & $100,0 \%$ \\
\hline \multicolumn{16}{|c|}{ Nordeste } \\
\hline BA & 4.390 & 1.442 & 189 & 1.268 & 51 & 2.510 & 834 & 141 & 598 & 17 & 678 & 27 & 149 & $59,6 \%$ & $63,0 \%$ \\
\hline CE & 3.098 & 791 & 142 & 831 & 53 & 2.075 & 456 & 97 & 553 & 21 & 614 & 21 & 79 & $71,2 \%$ & $100,0 \%$ \\
\hline PE & 3.830 & 1.394 & 191 & 982 & 42 & 1.923 & 781 & 118 & 396 & 20 & 595 & 15 & 77 & $80,3 \%$ & $100,0 \%$ \\
\hline PI & 1.018 & 245 & 56 & 335 & 14 & 716 & 161 & 22 & 281 & 5 & 301 & 10 & 50 & $96,7 \%$ & $100,0 \%$ \\
\hline RN & 1.290 & 419 & 52 & 443 & 12 & 747 & 217 & 33 & 232 & 5 & 237 & 7 & 40 & $89,5 \%$ & $100,0 \%$ \\
\hline MA & 1.833 & 563 & 84 & 453 & 2 & 1.047 & 303 & 52 & 178 & 0 & 218 & & 107 & $62,4 \%$ & \\
\hline $\mathrm{AL}$ & 994 & 265 & 56 & 269 & 5 & 623 & 176 & 41 & 130 & 5 & 204 & 5 & 30 & $52,9 \%$ & $0,0 \%$ \\
\hline
\end{tabular}


Quadro 1. (cont.)

\begin{tabular}{|c|c|c|c|c|c|c|c|c|c|c|c|c|c|c|c|}
\hline \multirow[b]{2}{*}{ UF } & \multicolumn{5}{|c|}{ Total de leitos (Lt) cadastrados CNES } & \multicolumn{5}{|c|}{ Leitos (Lt) SUS } & \multicolumn{2}{|c|}{ Leitos habilitados Covid* } & \multicolumn{3}{|c|}{ Hospitais Operantes } \\
\hline & $\begin{array}{l}\text { Lt. Comple- } \\
\text { mentares } \\
\text { existentes }\end{array}$ & $\begin{array}{l}\text { Lt. UTI } \\
\text { adulto }\end{array}$ & $\begin{array}{l}\text { Lt. UTI } \\
\text { pediátrica }\end{array}$ & $\begin{array}{l}\text { Lt. UTI } \\
\text { Covid } \\
\text { adulto }\end{array}$ & $\begin{array}{l}\text { Lt. UTI } \\
\text { Covid } \\
\text { pediátrico }\end{array}$ & $\begin{array}{l}\text { Lt. Com- } \\
\text { plementa- } \\
\text { res SUS }\end{array}$ & $\begin{array}{l}\text { SUS_UTI } \\
\text { adulto }\end{array}$ & $\begin{array}{l}\text { SUS_UTI } \\
\text { pediátrica }\end{array}$ & $\begin{array}{l}\text { SUS_UTI } \\
\text { Covid-19 } \\
\text { adulto }\end{array}$ & $\begin{array}{l}\text { SUS_UTI } \\
\text { Covid-19 } \\
\text { pediátrico }\end{array}$ & $\begin{array}{l}\text { Lt. habili- } \\
\text { tado Covid } \\
\text { UTI adulto }\end{array}$ & $\begin{array}{l}\text { Lt. habilitado } \\
\text { Covid UTI } \\
\text { pediátrico }\end{array}$ & $\begin{array}{l}\text { № de } \\
\text { hospitais } \\
\text { operantes }\end{array}$ & $\begin{array}{l}\text { \% Lt. Covid } \\
\text { habilitado } \\
\text { adulto }\end{array}$ & $\begin{array}{l}\text { \% Lt. Covid } \\
\text { habilitado } \\
\text { pediátrico }\end{array}$ \\
\hline \multicolumn{16}{|c|}{ Nordeste } \\
\hline PB & 1.278 & 450 & 108 & 249 & 7 & 902 & 300 & 76 & 164 & 6 & 196 & & 39 & $55,1 \%$ & \\
\hline SE & 802 & 248 & 22 & 169 & 7 & 398 & 151 & 20 & 44 & 0 & 136 & & 19 & $58,8 \%$ & \\
\hline \multicolumn{16}{|l|}{ Sul } \\
\hline RS & 4.328 & 1.668 & 251 & 917 & 10 & 2.849 & 985 & 178 & 672 & 5 & 704 & 5 & 167 & $93,3 \%$ & $100,0 \%$ \\
\hline$P R$ & 4.602 & 2.052 & 241 & 886 & 53 & 2.910 & 1.218 & 172 & 585 & 35 & 637 & 35 & 126 & $89,6 \%$ & $85,7 \%$ \\
\hline SC & 2.595 & 857 & 127 & 822 & 42 & 1.549 & 543 & 87 & 512 & 11 & 549 & 31 & 84 & $100,0 \%$ & $100,0 \%$ \\
\hline \multicolumn{16}{|c|}{ Sudeste } \\
\hline $\mathrm{SP}$ & 21.885 & 8.378 & 1.475 & 5.185 & 147 & 10.140 & 3.575 & 727 & 2.425 & 0 & 2.905 & 4 & 409 & $85,4 \%$ & $100,0 \%$ \\
\hline$M G$ & 7.996 & 3.174 & 348 & 2.159 & 30 & 4.718 & 2.027 & 194 & 927 & 0 & 1.120 & & 285 & $92,0 \%$ & \\
\hline RJ & 10.697 & 4.576 & 582 & 1.946 & 65 & 3.481 & 1.152 & 144 & 739 & 16 & 848 & 16 & 180 & $76,8 \%$ & $100,0 \%$ \\
\hline ES & 2.120 & 769 & 90 & 608 & 27 & 1.284 & 390 & 50 & 541 & 0 & 541 & & 46 & $74,5 \%$ & \\
\hline Brasil & 86.392 & 31.940 & 4.938 & 20.203 & 729 & 44.576 & 15.322 & 2.669 & 10.228 & 200 & 12.244 & 249 & 2.278 & $79,7 \%$ & $80,7 \%$ \\
\hline
\end{tabular}

Fonte: Elaboração própria

*Leitos Habilitados Covid - dados publicados até 31/08/2020.

\section{Iniciativas para o cálculo de necessidades de leitos de UTI na pandemia}

Em um cenário de insuficiência e má distribuição de leitos de UTI para enfrentamento da Covid-19 e insuficiência no volume de recursos financeiros despendidos, diversos modelos matemáticos foram construídos, desde o início da pandemia, para estimar a necessidade de recursos diversos para o atendimento hospitalar. Muitos deles centraram suas previsões no número de leitos de enfermaria e de UTI para atender os pacientes mais graves.

Para o Brasil, pelo menos três modelos foram desenvolvidos. Os autores deste artigo participaram do desenvolvimento de um deles, em uma reunião de esforços de pesquisadores do Núcleo de Educação em Saúde Coletiva (Nescon), órgão da Faculdade de Medicina da Universidade Federal de Minas Gerais (UFMG), com professores do Departamento de Engenharia de Produção da Escola de Engenharia da mesma universidade, no âmbito do Laboratório de Tecnologias de Apoio à Decisão em Saúde (Labdec) ${ }^{\mathbf{1 8}}$.

O objetivo do trabalho foi propor um modelo matemático para previsão da disponibilidade de leitos durante a pandemia e calcular os momentos de ruptura dos sistemas, ou seja, quando faltariam leitos gerais e de UTI, baseado em diversas premissas, sendo que muitas delas podiam ser modificadas pelos gestores e profissionais da saúde para aproximar de situação específicas. A modelagem do fluxo interno de pacientes nos hospitais foi baseada no modelo proposto pela empresa Array Advisors e disponibilizado na Internet ${ }^{19}$, que utilizava uma simples curva de crescimento exponencial e que, à época, representaria de forma adequada o início da pandemia. $\mathrm{O}$ modelo Labdec foi ampliado com diversos ajustes e refinamentos, principalmente em relação às funções de previsão de demanda, em que foi utilizada uma função logística 
diferencial que tem uma boa capacidade de representar a evolução da curva epidêmica e fornecer as previsões quantitativas de infectados para o cálculo da demanda de leitos. Nesse modelo, a curva logística é ajustada dia a dia, por meio da entrada de dados dos últimos sete dias, permitindo uma maior aproximação à dinâmica da epidemia.

Desde o início da pandemia na China, observou-se que uma proporção próxima a 14\% do total de pessoas infectadas pela Covid-19 iria desenvolver formas mais graves e necessitaria de cuidados clínicos mais elaborados, inclusive de internações hospitalares. Desses pacientes, cerca de $6 \%$ seriam pacientes críticos que demandariam internação em UTI ${ }^{20}$. Na primeira fase de desenvolvimento do modelo Labdec, a adoção do pressuposto de que 'apenas' cerca de 1\% (cenário favorável) a $2 \%$ (cenário desfavorável) do total de casos desenvolveria formas mais graves da Covid-19 foi um de seus diferenciais. Esse percentual destoou completamente dos demais trabalhos nesse campo e dos modelos preditivos de casos, óbitos e leitos hospitalares que foram divulgados, nos quais se supôs que a totalidade da população iria adoecer em um curto período, resultando em previsões muito infladas quanto à necessidade de leitos. Em um segundo momento, foi desenvolvido um modelo mais complexo, de caráter epidemiológico, do tipo Seirs (Susceptible - Exposed - Infectious - Recovered - Susceptible), que permite simular inclusive o impacto de diferentes taxas de distanciamento social sobre a incidência da Covid-19, mais adequado para o monitoramento dos casos no prolongado período que se supõe e se seguirá à primeira 'onda' de casos.

A expectativa dos pesquisadores era de que, com a progressão da pandemia, dispusesse-se de informações detalhadas sobre o movimento dos pacientes nos leitos de UTI em todo o País, que permitiria realizar ajustes nas equações do modelo, aproximando-o melhor da realidade que buscava representar. Isso não se concretizou devido à inexistência de dados disponíveis praticamente. Apenas o estado do Ceará disponibilizou um banco de dados atualizados com todas as informações detalhadas por caso. O Censo Hospitalar específico para os leitos Covid criado pelo Ministério da Saúde, perante a frágil articulação com os estados e municípios, chegou a ser publicado em junho, porém com conteúdo inconsistente, facilmente observado pela simples comparação das taxas de ocupação das UTI de algumas capitais contidas em seus Boletins Epidemiológicos e as apresentadas pelo sistema, o que impossibilitou o seu uso.

\section{A retração da oferta e da demanda no contexto da pandemia}

Com a emergência da pandemia, muitos serviços foram suspensos ou tiveram sua produção muito reduzida, como foi o caso das cirurgias eletivas e significativa redução dos traumas, esta última certamente determinada pelo distanciamento social. Além disso, um grande contingente de usuários passou a justificar temor de procurar os serviços de saúde pelo elevado risco de contágio, o que provocou uma expressiva queda nas internações hospitalares em todas as clínicas.

No seu conjunto, as internações caíram $10,1 \%$ em março, $35,9 \%$ em abril e $40,2 \%$ em maio em relação a janeiro de 2020 . As internações para procedimentos clínicos reduziram-se em 7,7\% em março, 28,8\% em abril e 33,4\% em maio, e os procedimentos cirúrgicos sofreram queda ainda maiores, de $13,4 \%, 45,7 \%$ e $49,5 \%$ respectivamente. Os transplantes foram ainda mais afetados, com expressivas e preocupantes quedas de $19,0 \%, 49,5 \%$ e $76,1 \%$ respectivamente. As maiores quedas, no entanto, deram-se no grupo de procedimentos 02 - Procedimentos com Finalidade Diagnóstica, que apresentou produções $15,6 \% ; 56,1 \%$ e $54,6 \%$ inferiores ao mês de referência, nos três meses analisados. Esses dados podem ser observados na tabela 1. 
E uma análise mais desagregada, observa-se que, em uma classificação que agrupa uma grande proporção de traumatismos diversos, como o subgrupo 0308, “Tratamento de lesões, envenenamentos e outros, decorrentes de causas externas', houve expressiva redução de internações, com quedas de $8,9 \%, 25,9 \%$, $30,7 \%$ e $40,1 \%$ nos meses de março a junho em relação a janeiro de 2020.

Tabela 1. Crescimento percentual da quantidade de internações hospitalares, Brasil, janeiro a junho de 2020. Mês base: janeiro de 2020

\begin{tabular}{|c|c|c|c|c|c|c|}
\hline Grupos de procedimento & 2020/Jan & 2020/Fev & 2020/Mar & $2020 / \mathrm{Abr}$ & 2020/Mai & 2020/Jun \\
\hline 2 Procedimentos com finalidade diagnóstica & $0,0 \%$ & $-7,2 \%$ & $-15,6 \%$ & $-56,1 \%$ & $-54,6 \%$ & $-72,3 \%$ \\
\hline 3 Procedimentos clínicos & $0,0 \%$ & $-5,6 \%$ & $-7,7 \%$ & $-28,8 \%$ & $-33,4 \%$ & $-59,3 \%$ \\
\hline 4 Procedimentos cirúrgicos & $0,0 \%$ & $-8,0 \%$ & $-13,4 \%$ & $-45,7 \%$ & $-49,5 \%$ & $-66,9 \%$ \\
\hline 5 Transplantes de órgãos, tecidos e células & $0,0 \%$ & $-11,6 \%$ & $-19,0 \%$ & $-49,2 \%$ & $-56,1 \%$ & $-76,1 \%$ \\
\hline Total & $0,0 \%$ & $-6,6 \%$ & $-10,1 \%$ & $-35,9 \%$ & $-40,2 \%$ & $-62,6 \%$ \\
\hline
\end{tabular}

Fonte: Datasus/MS21

Na operação do sistema SIH/SUS, podem ser apresentadas para processamento AIH com datas de alta do paciente de até seis meses anteriores à competência do processamento 22 . Por exemplo, cerca de $47 \%$ do total das internações ocorridas em junho de 2019 foi registrado em até 10 meses posteriores, com internações remanescentes apresentadas ainda em abril de 2020. Reconhece-se, portanto, que os dados aqui apresentados ainda não se encontravam totalmente estabilizados, sendo essenciais retificações no futuro próximo, especialmente, os dados de junho de 2020, competência mais recente ao período de análise. Ainda que com essas limitações, julgou-se oportuna a apresentação dessas informações que apontam para a insofismável, abrupta e expressiva redução nas internações hospitalares nessa fase da pandemia.

Essa redução forçada nas internações implicará uma acumulação crescente de casos, que, somada à histórica demanda reprimida de cirurgias eletivas, poderá resultar em um contexto potencialmente explosivo, agravado pela redução no aporte de recursos federais para o SUS, se aprovado o Projeto de Lei de Diretrizes Orçamentárias (PLDO) de 2021. Essa perda, segundo a Comissão Intersetorial de Orçamento e Financiamento do Conselho Nacional de Saúde (CNS), representaria uma subtração de R $\$ 35$ bilhões, na comparação com o montante de recursos orçamentários destinados ao Ministério da Saúde em $2020^{23}$.

\section{Uma agenda para o setor hospitalar}

Dadas todas as fragilidades e incertezas apresentadas, há que se indicar, ao menos inicialmente, proposições ao campo político, econômico e na organização dos serviços de saúde para seu enfrentamento, manifestadas a partir de algumas diretrizes para a reorganização hospitalar no País, com vistas a impactar nos determinantes estruturais e conjunturais da crise assistencial suscitados ou agudizados pela pandemia.

\section{Modalidades de financiamento e sua suficiência}

A crise assistencial nunca será superada se não houver uma total revisão na política de financiamento dos serviços de saúde públicos 
e contratados no Brasil. A primeira e fundamental providência seria a imediata revogação da Emenda Constitucional no 95 , que 'congelou' os recursos da área social por 20 anos. Não se pode restringir gastos em um sistema de saúde que se pretende universal e que está operando a partir de unidades assistenciais em franca situação de insolvência financeira já instalada há vários anos. As políticas de ajuste fiscal, além de terem se mostrado ineficazes para reverter a recessão crônica, redundam na elevação da pobreza, do desemprego e do endividamento das famílias, aumentando o adoecimento e a mortalidade das pessoas mais fragilizadas. Essas políticas, portanto, de forma contraditória, induzem aumento da demanda ao mesmo sistema de saúde que as restrições financeiras delas resultantes vem minando com grande sucesso.

Apesar dos interesses de setores empresariais da área da saúde apoiados por governos austeros em fomentar social e financeiramente o direcionamento de usuários para o setor de saúde suplementar no Brasil, a Covid-19 também põe em xeque esses interesses. As operadoras de Planos e Seguros Privados de Saúde passam a sofrer com as possibilidades de inadimplência dos assegurados e/ou redução considerável dessa carteira de clientes. Já os hospitais filantrópicos e privados, ao se basear majoritariamente na modalidade de pagamento por serviços profissionais ('fee for service'), encontram-se, no momento, ameaçados de colapso financeiro, com a redução da demanda e da produção de procedimentos assistenciais no decurso da pandemia. Essa redução não significa diminuição de custos fixos tampouco dos custos variáveis. Os custos fixos de manutenção da estrutura predial, assistencial e administrativa, em especial da força de trabalho, mantêm-se em uma proporção que pode ser estimada grosseiramente entre $70 \% \mathrm{e}$ $85 \%$ dos custos totais ${ }^{24}$. Já os custos variáveis ganham notadamente maior incerteza, seja pela inflação de preços provocada pela escassez relativa de insumos, materiais e equipamentos hospitalares, seja pelos custos adicionais decorrentes da ampliação e variação remuneratória das equipes médicas e de enfermagem para o atendimento dos casos com Covid-19. Note-se que nenhum desses dois fatores sofreu qualquer regulação específica nacional, demonstrando mais uma dimensão da fragilidade da coordenação nacional das políticas de combate à pandemia.

Por outro lado, hospitais que vinham sendo remunerados por modalidades baseadas em orçamentação global, a exemplo dos filantrópicos 'contratualizados' por estados e municípios, parecem passar a gozar de situação financeira relativamente mais vantajosa que os serviços que se relacionam exclusivamente com pagadores privados.

Assim sendo, além de superar a anomia nacional na regulação de preços, tanto de insumos estratégicos quanto da força de trabalho em saúde, há que se reconfigurar as modalidades de remuneração dos serviços, seja pelo SUS, seja pelas operadoras, bem como assegurar volume de recursos condizentes com os custos reais da produção de serviços hospitalares.

As modalidades de remuneração emergentes, baseadas em 'valor', isto é, que miram nos benefícios efetivamente auferidos pelos pacientes na sua relação com os serviços de saúde, aliada à otimização do desempenho econômico da sua produção, repaginam as análises de custo-benefício e custo-efetividade das intervenções. Estas podem introduzir métricas de desempenho econômico no nível do cuidado individual e terão maior êxito de implementação se conjugada a aportes de recursos calculados com base no volume e qualidade global dos serviços. As pressões pela redução de custos e de aumento da segurança e qualidade dos serviços serão contrapostas à necessidade de garantir patamares mínimos de recursos aos prestadores de serviços, sob pena de se provocar sua insolvência financeira pela escalada de requisitos de toda ordem. 


\section{Bases regionalizadas de preços e valores de remuneração de serviços hospitalares}

Desde que surgiu, a famosa Tabela de Procedimentos do SUS vem tendo os seus valores questionados por todos. Estes são reconhecidamente inferiores aos custos operacionais para a realização dos procedimentos e atos profissionais que compõem a estrutura sob a qual ela foi elaborada. Sabe-se também que seus preços não refletem os valores reais pagos aos prestadores de serviço, a não ser em algumas áreas da chamada 'alta complexidade' (hemodiálises, cirurgias cardíacas, tratamentos oncológicos etc.). Ademais, aos valores da tabela, são acrescidos incentivos financeiros de toda ordem para uma parte significativa de hospitais da rede SUS, como, por exemplo, os incentivos das Redes Temáticas Prioritárias e os subsídios fiscais e acréscimos incorporados em contratos globais.

Esse complexo modelo de financiamento hospitalar do SUS não só impede a compreensão de seus critérios pela maioria dos leigos e afasta a cidadania de seu controle democrático como também reforça, frequentemente, a insistente reclamação dos prestadores quanto aos baixos valores da tabela SUS, mesmo quando esse referencial já foi abandonado há mais de 15 anos para a maioria dos hospitais contratados. A rigor, mesmo antes da 'contratualização' com valores globais de custeio iniciada em 2004, os chamados municípios em 'gestão plena do sistema' pelos critérios da Norma Operacional Básica do SUS 1996 (NOB 96) já podiam contratar hospitais privados com outros critérios que não estritamente os valores da tabela de procedimentos aplicados às metas contratuais, o que amplia o período dessas queixas mal direcionadas para quase um quarto de século.

Uma das formas de superar esses impasses seria a organização de um sistema de gestão de preços que pudesse definir, de forma legítima, multi-institucional e baseada em levantamentos regionalizados, os valores de referência para a compra de serviços dos prestadores públicos e privados. Não é possível que, em contextos epidemiológicos e de oferta de serviços de saúde tão díspares, conformando distintos 'mercados', com agentes econômicos praticando preços muito diferenciados, os serviços sejam remunerados com base em valores uniformes, contidos em uma tabela nacional. É fundamental que se desenvolvam dispositivos de apropriação e registro sistemático dos principais preços, coletados regionalmente e tratados para estabelecer referenciais regionais de modo legítimo e transparente. Isso evitaria a captura das gestões por interesses particularistas ou corporativos, principalmente se nesses novos arranjos institucionais fossem incluídos representantes de usuários, Ministério Público e respectivos Conselhos de Saúde, garantindo-se seu caráter público e o interesse comum.

\section{Definição nacional de uma tipologia hospitalar}

O movimento de descentralização do SUS, alicerçado na legislação que regulamentou o setor, ampliou consideravelmente o poder de estados e municípios na definição de diversos aspectos da organização dos seus sistemas de saúde. Apesar da existência de uma norma ministerial que estabelece uma 'classificação hospitalar' ${ }^{25}$, as tipologias definidas não foram incorporadas ou mesmo adaptadas na maioria dos estados para orientar os investimentos federais na construção ou expansão de serviços hospitalares. Alguns estados, no entanto, instituíram tipologias hospitalares para ordenar o desenho de suas redes de atenção, como foi o caso dos estados da Bahia e de Minas Gerais.

No âmbito das chamadas 'Redes Temáticas', foram definidos requisitos para a classificação dos hospitais em algumas das redes, como foi o caso da Rede de Atenção às Urgências \& Emergências (RUE). Porém, contraditoriamente, manteve-se sobreposição de diferentes classificações sobre 
o mesmo conjunto de serviços, como é o caso da 'Linha de Cuidado de Atenção ao Trauma', que exige atributos não necessariamente coerentes com os definidos para as unidades que compõem a RUE.

Propõe-se, portanto, a definição de uma tipologia de hospitais que possa alicerçar um movimento de investimentos racionalmente direcionados à construção de uma rede de serviços equanimemente distribuída no território, fixando-se tamanhos de populações a serem abrangidas, distâncias/tempos de deslocamento máximos a serem percorridos pelos usuários e demais critérios de incorporação tecnológica e de recursos humanos especializados para cada nível de atenção.

\section{Criação de Complexos Hospitalares}

O grande número de hospitais com baixo número de leitos no Brasil (Hospitais de Pequeno Porte - HPP) tem sido um problema recorrente, tanto para a sociedade quanto para a gestão ${ }^{26}$. Se para muitos brasileiros esses serviços podem ser a única oportunidade de acesso, mesmo sem necessariamente garantir padrões mínimos de qualidade e efetividade, para a gestão, colocam-se os desafios da manutenção da força de trabalho assistencial necessária e o seu custo relativamente elevado ante a baixa capacidade fiscal da maioria dos pequenos municípios nos quais esses hospitais se encontram.

A solução simplista do fechamento puro e simples dos pequenos hospitais condenaria um grande número de pessoas à desassistência e não é abraçada pelas lideranças políticas de todos os níveis. A sua permanência no cenário assistencial por décadas, apesar do pesado ataque a que têm sido submetidos, cobra explicações, atitudes e análises técnicas muito mais elaboradas do que apenas a constatação de sua baixa escala econômica, suas baixíssimas taxas de ocupação e qualidade questionável.

Uma das soluções possíveis para aumentar os níveis de utilização dos HPP e ampliar os ganhos de escala do conjunto dos leitos hospitalares seria a organização de Complexos Hospitalares, aqui entendidos como arranjos institucionais que, mesmo preservando o status jurídico e a autonomia administrativa de cada uma das entidades envolvidas e seus corpos diretivos e técnicos, promoveria alianças estratégicas com vistas à otimização da capacidade instalada, reorientação da missão assistencial e do fluxo de pacientes. Os HPP passariam a representar unidades descentralizadas de Complexos Hospitalares destinadas à continuidade do cuidado de pacientes atendidos nos hospitais maiores, quando da possibilidade de transferência para estabelecimentos com menor densidade tecnológica, a exemplo dos casos de reabilitação e recuperação fortemente dependentes de cuidados de enfermagem e reabilitação e/ou para pacientes em cuidado paliativo.

Os recursos financeiros de custeio e investimento de cada ente seriam objeto de planejamento regional integrado sem necessariamente compor um fundo único, mas orientando a destinação dos recursos inseridos nos orçamentos de cada uma das entidades envolvidas a partir de decisões de caráter sistêmico entre os seus entes, com foco na garantia da integralidade e na progressividade do cuidado. Já os hospitais maiores deveriam garantir retaguarda assistencial e promoção da qualificação profissional tanto assistencial quanto gerencial de todo o corpo de recursos humanos envolvido, em uma relação de corresponsabilidade e cooperação técnica.

Para readequação das unidades e promoção da parceria, gestores municipais, estaduais e federais deveriam repensar processos regulatórios assistenciais, fomentar infraestrutura de tecnologia de comunicação e informação para implantação e compartilhamento de Prontuários Eletrônicos dos Pacientes e de Planos Terapêuticos Individuais (PTI), bem como garantir rede de apoio diagnóstico e de transporte sanitário. Ademais, a gestão do sistema de saúde também precisaria aperfeiçoar critérios de controle e monitoramento da integração regional. 


\section{Ampliação da rede hospitalar e manutenção dos leitos de UTI criados na pandemia}

Os leitos de UTI abertos para o enfrentamento da Covid-19 podem ser mantidos em sua totalidade, permanecendo como leitos de UTI ou convertendo-os em leitos de cuidados semi-intensivos e/ou de cuidado progressivo. Estes últimos são pouco utilizados no País, a não ser no caso da atenção neonatal, regulamentados no âmbito da Rede de Atenção à Gravidez, Parto e Puerpério (Rede Cegonha). O cuidado intermediário a adultos, apesar de duas consultas públicas realizadas sobre o tema, não tem suas normas de estrutura e funcionamento definidas, muito menos um financiamento específico disponível. O número desses leitos, aceitando-se a experiência dos países europeus, seria aproximadamente equivalente ao de leitos de UTI Adulto convencionais ${ }^{27}$.

\section{Conclusões}

A pandemia da Covid-19 trouxe à tona as contradições da oferta de serviços de saúde públicos e privados no País. Apesar do subfinanciamento crônico no caso do setor público, agravado por um longo período de estagnação econômica, os gestores conseguiram ampliar, emergencialmente e de forma muito expressiva, as estruturas assistenciais necessárias, como tem sido o caso dos leitos de UTI.

O atendimento dos casos de Covid-19 em uma rede de serviços com graves distorções na alocação geográfica dos recursos assistenciais, inclusive de leitos de UTI, tem tensionado enormemente o sistema. No entanto, uma maior valorização do sistema público de saúde por parte da população poderá, na dependência do andamento da crise política e econômica - e, principalmente, das alternativas que forem adotadas, de forma planejada ou não -, trazer um novo alento ao processo de consolidação do SUS.
Por outro lado, pressões oriundas do setor privado podem neutralizar esses ganhos, ao premerem pela transferência de recursos públicos para amortecer as grandes perdas do setor lucrativo, resultantes, principalmente, da mudança dos padrões de utilização dos serviços. Se essas propostas já frequentavam a agenda setorial nos últimos anos, certamente serão retomadas pelos interesses capitalistas do setor.

As mudanças dos padrões de utilização dos serviços são reflexo dos novos comportamentos sociais que, consequentemente, suscitam novas demandas ao sistema de saúde, seja ele público ou privado. Não se sabe ao certo nem quando nem como essas mudanças, de fato, incidirão. O que se pode antever é que a demanda por serviços hospitalares retraída no período mais crítico da pandemia poderá, no mínimo, provocar novas crises no sistema hospitalar, acrescentando ainda mais sofrimento e mortes evitáveis à contabilidade da pandemia.

Quaisquer que sejam os problemas, as propostas e as intenções, é fundamental o fortalecimento da gestão pública para que se garanta a suficiência de recursos humanos, de infraestrutura e de informação em saúde. Sem a busca de escala econômica adequada para os serviços de saúde, recursos de custeio garantidos para seu pleno funcionamento, transparência e corresponsabilidade na gestão, aliada a dispositivos institucionais eficazes de coordenação de políticas, nos espaços nacional, estadual e regional do sistema, envolvendo tanto a rede pública quanto a privada, não se pode aspirar, minimamente, a superação da crônica e danosa crise hospitalar, fortemente agudizada pela pandemia.

\section{Colaboradores}

Campos FCC (0000-0003-2172-0181)* e Canabrava CM (0000-0002-6906-3670)* contribuíram igualmente para a elaboração do manuscrito. 


\section{Referências}

1. Mckee M, Healy J. The role of the hospital in a changing environment. Bulletin of the World Health Organization. WHO. 2000; 78(6):803-810.

2. Organização Pan-americana da Saúde. A transformação da gestão de hospitais na América Latina e Caribe. Brasília, DF: OPAS;OMS; 2004.

3. De Negri Filho A. Bases para um debate sobre a reforma hospitalar do SUS: as necessidades sociais e o dimensionamento e tipologia de leitos hospitalares em um contexto de crise de acesso e qualidade [tese]. [São Paulo]: Universidade de São Paulo; 2016. 402 p.

4. Coelho IB. Os hospitais na reforma sanitária brasileira [tese]. [Ouro Preto]: Universidade Federal de Ouro Preto; 2013. 210 p.

5. Canabrava CM. Análise de implantação do programa de fortalecimento e melhoria da qualidade dos hospitais do sus de minas gerais - PRO-HOSP [tese]. [Salvador]: Instituto de Saúde Coletiva. Universidade Federal da Bahia; 2012. 330 p.

6. Jorge AO. A gestão hospitalar sob a perspectiva da micropolítica do trabalho vivo [tese]. [Belo Horizonte]: Universidade Federal de Minas Gerais; 2002. 225 p.

7. Agência Nacional de Vigilância Sanitária. Resolução $\mathrm{n}^{\circ} 7$, de 24 de fevereiro de 2010. Dispõe sobre os requisitos mínimos para funcionamento de Unidades de Terapia Intensiva e dá outras providências [internet]. [acesso em 2020 jun 22]. Disponível em: http:// bvsms.saude.gov.br/bvs/saudelegis/anvisa/2010/ res0007_24_02_2010.html.

8. Brasil. Emenda Constitucional no 95 , de 15 de dezembro de 2016. Altera o Ato das Disposições Constitucionais transitórias, para instituir o Novo regime Fiscal, e dá outras providências. Porto Alegre: Lex; 2016.

9. Brasil. Ministério da Economia. Subsecretaria de Política Econômica. Impactos Econômicos da COVID-19 [internet]. 2020. [acesso em 2020 jul 2]. Disponível em: http://www.fazenda.gov.br/centrais-de-conteudos/publicacoes/conjuntura-economica/estudos-economicos/2019/nota-impactos-economicos-da-covid-19.pdf/view.

10. Brasil. Medida Provisória $n^{\circ} 905$, de 11 de novembro de 2019. Institui o Contrato de Trabalho Verde e Amarelo, altera a legislação trabalhista, e dá outras providências [internet]. Diário Oficial da União. 12 Nov 2019 [acesso em 2020 jul 2]. Disponível em: http:// www.planalto.gov.br/ccivil_03/_ato2019-2022/2019/ Mpv/mpv905.htm.

11. Canabrava CM, Souza LEPF. A evolução da infraestrutura de sistema de saúde brasileiro: 2008-2017. In: Paim J, organizador. SUS - Tudo o que você precisa saber. São Paulo/Rio de Janeiro: Atheneu; 2019. p. 79-106.

12. Brasil. Ministério da Saúde. Portaria $n^{0} 1.646$, de 02 de outubro de 2015. Institui o Cadastro Nacional de Estabelecimentos de Saúde (CNES). Diário Oficial da União. 3 Out 2015.

13. Instituto Brasileiro de Geografia e Estatística. Estimativas da População: Tabelas [internet]. [acesso em 2020 jun 30]. Disponível em: https://www.ibge.gov. br/estatisticas/sociais/populacao/9103-estimativas-de-populacao.html?=\&t=downloads.

14. Agência Nacional de Saúde Suplementar. ANS disponibiliza número de beneficiários relativos a julho [internet]. [acesso em 2020 ago 26]. Disponível em: http://www.ans.gov.br/aans/noticias-ans/numeros-do-setor/5914-ans-disponibiliza-numeros-de-beneficiarios-relativos-a-julho.

15. Brasil. Ministério da Saúde. Portaria $n^{\circ} 568$, de 26 de março de 2020. Autoriza a habilitação de leitos de Unidade de Terapia Intensiva Adulto e Pediátrica para atendimento exclusivo dos pacientes com a COVID-19. Diário Oficial da União. 8 Abr 2020.

16. Grupo IAG Saúde. Estimativa de Custos Operacionais dos leitos de UTI Adulto em consequência do 
Covid-19. [acesso em 2020 jun 22]. Disponível em: https://www.drgbrasil.com.br/valoremsaude/estimativa-de-custos-operacionais-dos-leitos-de-uti-adulto-em-consequencia-do-covid-19/.

17. Grupo IAG Saúde. Saúde pública e privada: os caminhos para a sustentabilidade das redes hospitalares na pandemia [Vídeo conferência]. [acesso em 2020 jun 22]. Disponível em: https://www.youtube.com/ watch?v=Tge 2 u3EU7io.

18. Universidade Federal de Minas Gerais, Laboratório de Tecnologias de Apoio à Decisão em Saúde. Simulador Leitos COVID-19 [internet]. [acesso em 2020 jun 18]. Disponível em: https://labdec.nescon.medicina.ufmg.br/

19. Array A. Array Advisors' Model Validates Fears of ICU Bed Shortage Due to Coronavirus Pandemic [internet]. [acesso em 2020 jun 22]. Disponível em: https://array-architects.com/press-release/array-advisors-model-validates-fears-of-icu-bed-shortage-due-to-coronavirus-pandemic/.

20. World Health Organization. Report of the WHO-China Joint Mission on Coronavirus Disease 2019 (CO VID-19) [internet]. 2020. [acesso em 2020 jun 30]. Disponível em: https://www.who.int/docs/default-source/coronaviruse/who-china-joint-mission-on-covid-19-final-report.pdf.

21. Brasil. Ministério da Saúde, Datasus. Internações hospitalares por área [internet]. 2020 [acesso em 2020 jul 13]. Disponível em: http://tabnet.datasus.gov.br/ cgi/tabcgi.exe?sih/cnv/sxuf.def.

22. Brasil. Ministério da Saúde, Secretaria de Atenção à Saúde, Departamento de Regulação, Avaliação e Controle. Manual técnico do Sistema de Informação Hospitalar [internet]. Brasília, DF: Editora do
Ministério da Saúde; 2007. [acesso em 2020 ago 26]. Disponível em: http://bvsms.saude.gov.br/bvs/ publicacoes/07_0066_M.pdf.

23. Brasil. Ministério da Saúde, Conselho Nacional de Saúde. Petição Pública: você vai deixar o SUS perder mais R $\$ 35$ bilhões em 2021? [internet]. 2020. [acesso em 2020 ago 25]. Disponível em: http://www.conselho.saude.gov.br/ultimas-noticias-cns/1297-peticao-publica-voce-vai-deixar-o-sus-perder-mais-r-35-bilhoes-em-2021.

24. La Forgia GM, Couttolenc BF. Desempenho hospitalar no Brasil: em busca da Excelência. São Paulo: Singular; 2009.

25. Brasil. Ministério da Saúde. Portaria no 2.224, de 05 de dezembro de 2002. Estabelece o sistema de Classificação Hospitalar do Sistema Público de Saúde [internet]. [acesso em $2020 \mathrm{dez}$ 6]. Disponível em: http://www.sbccv.org.br/medica2-old/downloads/ Portaria\%20n\%C2\%BA\%202.224-GM\%20Em\%20 5\%20de\%20dezembro\%20de\%202002.pdf.

26. Carmo M. Hospitais de pequeno porte e rede de atenção à saúde: um estudo de sua inserção e avaliação em Minas Gerais no período 2004/2014 [tese]. [Belo Horizonte]: Universidade Federal de Minas Gerais; 2016. 207 p.

27. Rhodes A, Ferdinande $\mathrm{P}$, Flaatten $\mathrm{H}$, et al. The variability of critical care bed numbers in Europe. Intensive Care Med [internet]. 2012. [acesso em 2020 jun 23]; 38(10):1647-1653. Disponível em: https://link. springer.com/article/10.1007/s00134-012-2627-8.

Recebido em 03/07/2020

Aprovado em 18/09/2020

Conflitos de interesses: inexistente

Suporte financeiro: não houve 